Intersections

Canadian Journal of Music

Revue canadienne de musique
Intersections CANADAN TOURAA OP NUSIC

Emmanuel Reibel. 2013. Comment la musique est devenue

" romantique ": De Rousseau à Berlioz. Paris : Fayard, coll.

"Les chemins de la musique ».463 p. ISBN 978-2-213-67849-8

\title{
Bruno Coulombe
}

Volume 34, numéro 1-2, 2014

URI : https://id.erudit.org/iderudit/1030876ar

DOI : https://doi.org/10.7202/1030876ar

Aller au sommaire du numéro

Éditeur(s)

Canadian University Music Society / Société de musique des universités

canadiennes

ISSN

1911-0146 (imprimé)

1918-512X (numérique)

Découvrir la revue

Citer ce compte rendu

Coulombe, B. (2014). Compte rendu de [Emmanuel Reibel. 2013. Comment la musique est devenue " romantique" : De Rousseau à Berlioz. Paris : Fayard, coll. "Les chemins de la musique ». 463 p. ISBN 978-2-213-67849-8]. Intersections, 34(1-2), 186-190. https://doi.org/10.7202/1030876ar

Copyright (c) Canadian University Music Society / Société de musique des universités canadiennes, 2015
Ce document est protégé par la loi sur le droit d'auteur. L'utilisation des services d’Érudit (y compris la reproduction) est assujettie à sa politique d'utilisation que vous pouvez consulter en ligne.

https://apropos.erudit.org/fr/usagers/politique-dutilisation/ 
Emmanuel Reibel. 2013. Comment la musique est devenue «romantique»: De Rousseau à Berlioz. Paris : Fayard, coll. «Les chemins de la musique». 463 p. ISBN 978-2-213-67849-8.

«Le romantisme n'est pas un style mais un projet $^{1}$ », a déjà déclaré le pianiste et musicologue Charles Rosen, auteur de l'ouvrage La génération romantique (1995), dans lequel il s'attardait à décrire les innovations de langage héritées du romantisme en musique, à partir notamment des ouvres d'Hector Berlioz (1803-1869), de Frédéric Chopin (1810-1849) ou de Franz Liszt (1811-1886). Cette définition, aussi incomplète soit-elle, nous laisse néanmoins entrevoir le caractère hétérogène de la musique dite «romantique», qui ne saurait donc se ramener à un style, à une période ni même à une essence précise, qu'il serait possible de situer dans le temps ou chez certains compositeurs en particulier. Mais comment le terme "romantique», d'abord issu du langage pictural, en est-il venu à désigner tout un pan de la musique instrumentale et vocale du $\mathrm{XIX}^{\mathrm{e}}$ siècle, et même jusqu'au début des années 1900 si nous considérons les œuvres d'un Sergueï Rachmaninov (1873-1943), par exemple? Car si l'adjectif est aujourd'hui couramment employé pour décrire une musique qui se distingue par sa sensibilité, sa fièvre lyrique et son expression de sentiments exacerbés, il n'a pas toujours eu cette connotation.

Dans son ouvrage Comment la musique est devenue "romantique»: De Rousseau à Berlioz, Emmanuel Reibel s'attarde justement à reconstruire le sens du mot «romantique ( (ou devrions-nous plutôt dire «les sens »?) à partir des différents emplois du terme pour décrire la production musicale en France des années 1770 jusqu'à 1830, année qui marque la création de la Symphonie fantastique de Berlioz. D'entrée de jeu, l'auteur entend corriger ce qu'il considère comme certaines lacunes de l'historiographie musicale, notamment celle de considérer le romantisme comme une période et donc, de le circonscrire dans le temps, comme en font foi les travaux d'Alfred Einstein et de Leon Plantinga. Même Charles Rosen, que nous avons cité au début de cette recension, n'échappe pas aux récriminations de Reibel puisqu'il a choisi «de poser le romantisme comme une affaire historico-temporelle, générationnelle» (p. 17), mais sans en explorer toute l'épaisseur ou la diversité. Le terme "romantique» ne saurait non plus se ramener à une essence particulière, malgré les travaux marquants de Carl Dahlhaus qui a tenté de faire du romantisme plus qu'une simple expression de la sensibilité en proposant une approche musicologique héritée de la conception métaphysique de la musique d'E.T.A. Hoffmann. Ainsi, le romantisme germanique, dans son essence, ne correspond pas au romantisme français, d'où la multiplicité de sens qui ont été accolés à ce mot dans les dernières décennies du XVIII siècle et la première moitié du XIX ${ }^{e}$. Et c'est là l'hypothèse de Reibel, à savoir que le romantisme musical, loin d'être un concept homogène, «s'est peu à peu construit, de façon différenciée suivant les pays et les courants de pensée, à partir de pratiques rédactionnelles diverses, au sein de différents écrits critiques, esthétiques, théoriques 
ou fictionnels» (p. 41). Pour l'auteur, le romantisme musical se situerait donc avant tout dans le champ du récepteur, avant d'investir l'œuvre en elle-même et éventuellement, le créateur.

Reibel distingue quatre grands paradigmes autour desquels la notion de romantisme s'est développée dans le discours musical. D’abord rattaché au concept de paysage, le terme «romantique» apparait pour la première fois en tant qu'adjectif dans Les Rêveries du promeneur solitaire, ouvrage inachevé de Rousseau rédigé entre 1776 et 1778 . Mais c'est Grétry qui est le premier à l'employer dans un sens musical dans ses Mémoires de 1789. À cette époque, la notion de romantisme désigne d'abord une musique dite «naturelle», plus proche du chant des oiseaux que de l'harmonie de la musique savante, tel que l'illustre l'air suisse du Ranz des vaches. Comme l'explique Reibel, la musique en vient à être considérée comme telle parce que le discours musical lui prête les mêmes attributs que le paysage romantique, à savoir sa dimension picturale, son aura poétique et sa vertu énergétique. L'objectif de cette musique est d'ailleurs de continuer à peindre, "même (surtout) lorsqu'elle est sans objet apparent ou référent explicite» (p. 74). Quelque 20 ans avant qu'E.T.A. Hoffmann ne consacre la musique comme "le plus romantique des arts», en raison justement de sa capacité à dépouiller l'auditeur «de tous les sentiments qu'on peut nommer pour plonger dans l'indicible ${ }^{2}$ ", la musique dite "romantique» entend donc, dès les années 1790, "se constituer comme image sonore dans l'esprit de l'auditeur» (p. 73). En s'éloignant de l'univers sensible, elle cherche avant tout à s'émanciper de tous les référents verbaux pour créer plutôt une impression vague que l'esprit pourra transformer en objet selon le procédé d'imitation.

En plus de son association avec la notion de paysage, le romantisme en vient de plus en plus à être considéré comme un signe du passé, ce que Reibel qualifie de paradigme temporel. Les deux idées sont liées, en ce sens que l'investissement du sujet dans les paysages observés active toujours la mémoire du contemplateur, d'où la naissance d'une certaine poétique du passé. C'est ainsi que la musique en vient à fonctionner «de plus en plus comme la contemplation des paysages, en impliquant un processus de distanciation temporelle» (p. 111). Mais, comme le démontre l'auteur, cette conception du romantisme en tant que souvenir ou fragment du passé se fait de manière un peu désordonnée dans le discours sur la musique en France. D’abord employé dans le même sens qu' «antique», l'adjectif «romantique», étroitement associé au genre de la romance, désigne tout autant les chants des bardes calédoniens que ceux des trouvères gothiques. Mais dans les deux cas, il sert à éveiller le souvenir d'un passé pittoresque et idéalisé, si bien que «la musique semble désormais l'un des meilleurs vecteurs pour annihiler le temps et réveiller les souvenirs» (p. 139). Cette association entre musique et passé dépasse le champ de la réception et du discours sur la musique puisque la nostalgie devient un principe d'écriture chez Grétry, qui sera le premier, dans son opéra Richard Cœur de Lion en 1784 , à utiliser la récurrence thématique pour instituer un certain rapport au

2 Hoffmann 1985 [1810], p. 38. 
temps dans une œuvre, procédé qui sera réutilisé par Carl Maria von Weber (1786-1826) et Berlioz.

Ce paradigme temporel associé au romantisme se double rapidement d'un paradigme géographique et national puisque, pour les Français, «ce qui réveille le souvenir de temps immémoriaux vient le plus souvent d'ailleurs» (p. 165). Ici, Reibel établit une distinction fondamentale entre la signification de l'adjectif «romantique» chez les Allemands et ce qu'il évoque en France. Dans la langue germanique, romantische fait référence à un type d'opéra-comique qui dépeint des sujets historiques ou légendaires en multipliant les invraisemblances, dans un registre qui allie romanesque, étrange, merveilleux et pittoresque. Mais le terme peine à s'imposer en France, où une vive polémique éclate entre ceux qui, comme madame de Staël ou Castil-Blaze, voudraient que les Français s'imprègnent de cette nouvelle pensée et ceux qui craignent une invasion étrangère, qu'elle soit issue d'Allemagne ou même d'Italie, avec un musicien comme Gioachino Rossini (1792-1868). C'est finalement l'association entre romantisme et merveilleux qui fait en sorte que l'adjectif est mieux accepté en France puisqu'il ne s'oppose plus directement à l'esprit du classicisme. D'abord utilisé pour décrire les contes d'Hoffmann et leur imagination créatrice, le terme «fantastique» en vient presque à être considéré comme un synonyme de «romantique» chez les Français, qui y voient une invitation à goûter «l'inquiétante étrangeté» (p. 240) d'une œuvre musicale.

Le quatrième paradigme décrit par Reibel en est un idéologique puisque l'évolution du discours sur la musique demeure indissociable des bouleversements politiques qui secouent la France à cette époque. En effet, la Révolution de 1789 et l'exécution de Louis XVI en 1793 ont laissé la France divisée en deux camps, les ultras et les libéraux, qui se positionneront tous deux à la fois en faveur et contre le romantisme. Empruntant la catégorisation de Paul Bénichou, Reibel distingue 1) les anti-romantiques ultras qui ne voient que déstabilisation de l'État derrière les réformes artistiques; 2) les anti-romantiques libéraux qui, fidèles à l'esprit des Lumières, se méfient du mysticisme et craignent un nouvel obscurantisme; 3 ) les romantiques ultras, qui en appellent à un renouveau spiritualiste et souhaitent faire de l'artiste le souverain des temps modernes; et 4) les romantiques libéraux, qui en appellent à la liberté de création et à une véritable révolution dans les arts. À travers ces quatre positionnements idéologiques, la figure d'Hector Berlioz émerge comme le plus ardent défenseur d'un romantisme libéral, lui qui «revendique la liberté de l'imagination créatrice, par-delà toutes les entraves institutionnelles ou scolastiques» (p. 282). Dans ce débat enflammé, quelques critiques tentent de proposer une esthétique du juste milieu, comme François-Joseph Fétis, le fondateur de la Revue musicale, qui prône la modération «dans la science des proportions, l'économie formelle et l'adéquation entre effets visés et moyens employés» (p. 308). S'il tente de relativiser l'originalité des compositeurs romantiques en faisant valoir que le «mouvement» existe depuis des siècles en musique, il refusera de condamner en bloc «les saisissantes innovations de la nouvelle école» (p. 308). 
Tout au long de l'ouvrage, Reibel prend bien soin d'établir des ponts entre les différents paradigmes de l'écoute romantique dans le but de montrer qu'ils n'évoluent pas en vase clos, mais qu'au contraire, ils interagissent et s'entrecroisent. C'est ainsi qu'autour de 1830, les différentes strates terminologiques associées au romantisme fusionnent pour lui donner enfin une apparence d'homogénéité. Plusieurs œuvres surgies à ce moment précis de l'histoire témoignent de cette stabilité esthétique. Créé en 1829, le dernier opéra de Rossini, Guillaume Tell, opère une véritable fusion des paradigmes romantiques, comme l'explique Reibel, avec son orchestre-paysage qui «conjugue évocation historique, assimilation française des styles étrangers et mise en valeur de la liberté sur le plan esthétique» (p. 332). Dans le genre symphonique, la Symphonie fantastique de Berlioz s'impose elle aussi comme une sorte d'archétype du romantisme, avec sa suite de tableaux poétiques, son thème de l'«idée fixe» qui entraîne l'auditeur dans un passé intra-diégétique, son association entre musique et étrangeté et la portée métaphoriquement "révolutionnaire» que le compositeur a voulu lui donner. Enfin, dans le répertoire pianistique, c'est l'Album d'un voyageur de Liszt qui fait fusionner les différentes modalités de l'écoute romantique, selon l'auteur, avec ses pièces qui allient nature et tableaux poétiques, méditations sur le temps qui passe, évocation d'inquiétantes étrangetés et esprit de synthèse entre romantisme libéral, révolution sociale et spiritualisme catholique.

Le mérite de Reibel est de montrer avec brio que la notion de romantisme reste irréductible à un style musical en particulier et qu'elle correspond avant tout à une façon particulière de dire et de concevoir la musique. Le terme, d'abord associé à la nature et au paysage, en est venu progressivement à qualifier des objets musicaux à la suite d'un transfert d'abord artistique (de la peinture à la musique), puis culturel (des pays britanniques et germaniques à la France), en plus d'avoir été influencé par les courants politiques et idéologiques. Il est également le fruit d'une stratification terminologique qui a vu plusieurs sens différents se combiner pour aboutir à une certaine conception du romantisme autour de 1830. L'approche de Reibel est multidisciplinaire et s'inscrit tout à fait en phase avec celle défendue par Carl Dahlhaus dans Fondements de l'histoire de la musique (1983), dans lequel il plaide pour une histoire structurelle de la musique qui tiendrait compte des liens de correspondance entre les facteurs économiques, sociaux, psychologiques, esthétiques et compositionnels pour expliquer l'émergence d'un style ou l'impact d'une œuvre. En même temps, bien qu'il s'intéresse avant tout à la réception des œuvres, Reibel ne néglige pas pour autant la dimension poiétique en montrant comment certains compositeurs ont cherché à construire une écoute "romantique " par l'utilisation de divers effets, rejoignant ainsi la pensée de Leo Treitler, pour qui «l'histoire de la musique n'est possible que si l'historien sait montrer la place occupée par les œuvres dans l'histoire en révélant la part d'histoire qu'elles contiennent ${ }^{4}$ ». L'objectif de Reibel n'est certes pas de restreindre l'emploi du

4 « Music history is possible only insofar as the historian is able to show the place of individual works in history by revealing the history contained within the work themselves " (Trietler 1989, p. 173). 
mot romantisme, au contraire, mais de montrer qu'il «participe toujours d'une construction esthétique ou idéologique, interprétant la musique et façonnant son histoire» (p. 373).

\section{RÉFÉRENCES}

Bénichou, Paul. 1973. Le Sacre de l'écrivain, 1750-1830. Essai sur l'avènement d'un pouvoir spirituel läque dans la France moderne. Paris : Librairie J. Corti.

Dahlhaus, Carl. 2013. Fondements de l'histoire de la musique, traduit de l'allemand par Marie-Hélène Benoît-Otis. Arles : Actes Sud/Cité de la musique.

___.1997. L'idée de la musique absolue : une esthétique de la musique romantique, traduit de l'allemand par Martin Kaltenecker. Genève: Contrechamps. Einstein, Alfred. 1959. Music in the Romantic Era. New York: W.W. Norton.

Hoffmann, E.T.A. 1985 [1810]. «Ludwig van Beethoven, Cinquième Symphonie (Avril-Mai 1810)», Écrits sur la musique, traduit de l'allemand par Brigitte Hébert et Alain Montandon, 38-51. Lausanne : L'Âge d'homme.

Plantinga, Leon. 1989. La musique romantique. Histoire du style musical au $X I X^{e}$ siècle en Europe, traduit de l'américain par Dennis Collins. Paris : J.C. Lattès.

Rosen, Charles. 2002 [1995]. La génération romantique: Chopin, Schumann, Liszt et leurs contemporains, traduit de l'anglais par Georges Bloch. Paris : Gallimard.

_. 1993. Plaisir de jouer, plaisir de penser : conversation avec Catherine Temerson. Paris : Eshel, Genève : Georg.

Treitler, Leo. 1989. Music and the Historical Imagination. Cambridge : Harvard University Press.

Bruno Coulombe

Élizabeth Gallat-Morin. 2012. L'orgue de 1753 renaît de ses cendres. Québec : Musée de la civilisation, 96 p. ISBN 978-2-551-25342-5 (couverture souple).

Tous les instruments musicaux fabriqués de main d'homme, que ce soit par un facteur ou par un luthier sont, par définition, uniques. De ceux-ci, l'orgue est probablement l'instrument dont les traits distinctifs sont les plus marqués d'un modèle à l'autre. En effet, les éléments constitutifs de l'orgue, par exemple la forme de son buffet, le nombre de ses claviers ou encore le choix des jeux qui le composent, sont propres à chaque instrument.

Dans le même ordre d'idées, chaque orgue est chargé d'une histoire elle aussi unique, tributaire des circonstances particulières liées à sa commande, à sa fabrication, à son installation et à son utilisation à une époque et dans un lieu donnés. Élisabeth Gallat-Morin nous livre l'un de ces récits dans son ouvrage intitulé L'orgue de 1753 renaît de ses cendres, qui se révèle, au fil des pages, être une véritable épopée. 